\title{
Sociodemographic profile of Infertile Women Presenting at Bangabandhu Sheikh Mujib Medical University
}

\author{
S Ishrat ${ }^{1}, \mathrm{~F} \mathrm{Deeba}^{2}$, P Fatima $^{3}$
}

\begin{abstract}
Background: Infertility is a medical as well as a psychological problem of the couples who suffer from it. To serve them efficiently, their sociodemographic profile must be taken into consideration during work up, treatment and counseling. Objective: The objective of the study was to describe and analyze the sociodemographic profile of the infertile women attending Bangabandhu Sheikh Mujib Medical University. Method: Descriptive analysis of data collected and compiled by interviewing the couples attending Infertility outdoor of Bangabandhu Sheikh Mujib Medical University. Results: $64.64 \%$ of the women were in the age group $20-29$ years. $64.13 \%$ of women had 10 or less than 10 years of education. $79.79 \%$ of women were housewives.69.69\%) of women were from urban areas, with $59.06 \%$ from within Dhaka city. $35.35 \%$ of the women were from low and $37.37 \%$ of the women were from middle socioeconomic condition. One third of women presented with less than three years of infertility. The ratio of primary and secondary infertility is 2:1. Badly managed labour and delivery was the most common cause of secondary infertility. Conclusion: The findings of the study help us understand the needs and abilities of the infertile women presenting to us and decide the best possible strategies of treating and counseling them. [J Shaheed Suhrawardy Med Coll 2015;5(2): 63-65]
\end{abstract}

Keywords: Snfertility, Sociodemography

\begin{abstract}
Introduction
Infertility is as much a psychosocial crisis as it is a medical problem. Infertility can sometimes cause physical sufferings. Almost always it causes anxiety, depression, social isolation and sexual dysfunction ${ }^{1}$. The crisis has its own cultural, religious and class related aspects ${ }^{2}$. Infertility has many treatment options for its different causes. These options are bewildering for most of the couples, physically uncomfortable, costly and without a guarantee of success which make the condition particularly stressful ${ }^{1}$.

Bangabandhu Sheikh Mujib Medical University is one of the two highest public sector centres in the country receiving referral of patients with infertility. Infertility unit in the Department of Obstetrics \& Gynaecology offers comprehensive diagnostic and therapeutic services for the
\end{abstract}

infertile couples who cannot afford the expenses of private centres for infertility treatment.

Our study was on the socio-demographic profile of the infertile women or couples who attend our infertility unit at Bangabandhu Sheikh Mujib Medical University. The findings are expected to help us understand the needs and abilities of the people who come for our service and decide the best possible strategies of serving them.

\section{Method}

The study is a descriptive analysis of the data collected and compiled in the Infertility outdoor of Bangabandhu Sheikh Mujib Medical University. In the year of 2012 a total of 2810 couples attended the Infertility outdoor for the first time. With a prevalence of infertility at $15 \%$, the sample size was

1. Shakeela Ishrat, Assistant Professor, Infertility unit, Department of Obstetrics \& Gynaecology, Bangabandhu Shekh Mujib Medical University 2. Farzana Deeba, Assistant Professor, Infertility unit, Department of Obstetrics \& Gynaecology, Bangabandhu Shekh Mujib Medical University

3. Parveen Fatima, Professor, Infertility unit, Department of Obstetrics \& Gynaecology, Bangabandhu Shekh Mujib Medical University

\section{Correspondence}

Dr. MHM Alamgir, Assistant Professor, Department of Orthpaedic-Surgery, Shaheed Suhrawardy Medical. College, Sher-E-Bangla Nagar, Dhaka, Bangladesh; Email; d.malamgir@yahoo.com; Cell No.: +8801819144934

Contributions by authors: MHMA \& MI involved in protocol development, data collection, openative procedure as well as manuscript writings. MNI \& AK revised the manuscript 
estimated to be 192 . A total of 196 couples were selected by randomization. The collected data was analyzed manually. The women were categorized in different age groups namely 15-19 years, 20-29 years, 30-39 years and 40-45years. The socioeconomic condition was defined as low when monthly income was equal to or less than $10,000 \mathrm{Tk}$, medium when monthly income was more than $10,000 \mathrm{Tk}$ but less than $30,000 \mathrm{Tk}$ and high when monthly income was 30,000 Tk or more. The women were categorized as professional when they did some form of paid jobs or business outside home. Housewife or student is self explanatory. Regarding educational status, women were categorized into groups such as illiterate, education of 5 years or less, education of 6-10 years and education of more than 10 years.

Data was analyzed manually and organized in appropriate tables for interpretation.

\section{Results:}

A total of 196 women attending the Infertility outdoor were taken for study.

Table I shows the distribution of the women in different age, education, occupation and residence groups.

$64.64 \%$ of the women were in the age group 20-29 years. $64.13 \%$ of the women had 10 years or less than 10 years of education. Only $20.20 \%$ of women were students or professionals including teachers, nurses, garment workers, other small business or service. $79.80 \%$ of the women were housewives.

Looking at the residence of the women, $69.69 \%$ were from urban areas. $59.06 \%$ of the women were from within

Table I. Sociodemographic profile of infertile women

\begin{tabular}{lcc}
\hline Age (years) & No of women & Percentage of women \\
\hline $15-19$ & 07 & $3.53 \%$ \\
$20-29$ & 128 & $64.64 \%$ \\
$30-39$ & 60 & $30.30 \%$ \\
$40-45$ & 03 & $1.5 \%$ \\
\hline Education & No of women & Percentage of women \\
\hline Illiterate & 07 & $3.53 \%$ \\
$<5$ yrs education & 22 & $11.11 \%$ \\
$6-10$ yrs education & 98 & $49.49 \%$ \\
$>10$ yrs education & 71 & $35.85 \%$ \\
\hline Occupation & No of women & Percentage of women \\
\hline Students \& & 40 & $20.20 \%$ \\
professionals & & \\
Housewives & 156 & $79.79 \%$ \\
\hline Residence & No of women & Percentage of women \\
\hline Urban & 138 & $69.69 \%$ \\
Rural & 60 & $30.30 \%$ \\
Dhaka city & 111 & $56.06 \%$ \\
Dhaka District & 11 & $5.55 \%$ \\
outside Dhaka city & & $23.23 \%$ \\
Nearby districts & 46 & $14.64 \%$ \\
Remote districts & 29 &
\end{tabular}

Dhaka city, 23.23\% from nearby districts (less than 4 hours distance by road) and $14.64 \%$ from remote districts (more than 4 hours distance by road).

\section{Table II. Socioeconomic condition of infertile women}

\begin{tabular}{lcc}
\hline $\begin{array}{l}\text { Socioeconomic } \\
\text { condition (family } \\
\text { income per month) }\end{array}$ & No of women & Percentage of women \\
\hline$<10,000 \mathrm{Tk}$ & 70 & $35.35 \%$ \\
$>10,000-20,000 \mathrm{Tk}$ & 74 & $37.37 \%$ \\
$>20,000-30,000 \mathrm{Tk}$ & 38 & $19.19 \%$ \\
$>30,000 \mathrm{Tk}$ & 16 & $8.08 \%$ \\
\hline
\end{tabular}

Table II shows the socioeconomic condition of the women and her family. $35.35 \%$ of women were in low socioeconomic condition ( earn equal to or less than $10,000 \mathrm{Tk}$ per month), $37.37 \%$ were in middle socioeconomic condition ( earn more than 10,000 Tk but less than 30,000 Tk per month)., 8.08\% were in high socioeconomic condition ( earn $30,000 \mathrm{Tk}$ or more per month).

Table III. Duration and type of infertility

\begin{tabular}{lcc}
\hline Duration of infertility & No of women & Percentage of women \\
\hline$<3$ years & 60 & $30.30 \%$ \\
$3-10$ years & 119 & $60.40 \%$ \\
$>10$ years & 19 & $9.59 \%$ \\
\hline Type of infertility & No of women & Percentage of women \\
\hline Primary infertility & 124 & $62.94 \%$ \\
Secondary infertility & 73 & $37.05 \%$ \\
\hline
\end{tabular}

Table III shows duration and type of infertility. About duration of infertility $30.30 \%$ of women had less than 3 years of infertility and $60.40 \%$ had 3-10 years of Infertility. The ratio of primary and secondary infertility is almost 2:1 (124 vs 73). The most common (20.14\%) significant feature in obstetric history of women with secondary infertility appears to be puerperal sepsis with history of prolonged and obstructed labour and vaginal or caesarean delivery of stillborn babies. Other features are abortion (17.80\%), MR (7.6\%) and ectopic pregnancy $(4.1 \%)$.

\section{Discussion}

Our study shows that most of the women were in 20-29 years age group. This reflects the situation in Bangladesh where most of the women have early marriage and failure to conceive soon after marriage is a problem for them. This is different from the fertility centres in developed countries where most women present in fourth decade3. This is because in this countries, women tend to postpone their first pregnancy for education and carrier. Increased female age has a negative impact on fertility since the number and quality of oocyte decreases with age due to rising aneuploidy and the frequency of intercourse decreases after 30 years of age. Women presenting in our centre has the advantage of age unless they have gross 
male factor or tubal disorder.

Most (64.13\%) of the women had 10 yrs or less than 10 yrs of education. Most of the women were housewives. Housewives are lagging behind professionals in economic empowerment. Adequate and meaningful counseling is difficult with most of the women because of poor educational background. But a study in Srilanka ${ }^{4}$ had different findings. Women who were employed had higher rate of infertility compared to those who were not.

Most of the women had urban residence. Rural women were less in number though it is presumed that the problem of infertility is equally prevalent and sociocultural adversity of infertile women is more in rural region.

More than half of the infertile women were from within Dhaka city. It appears that the centre offers service mostly to the people of Dhaka city. $14.64 \%$ of the women come from the remote districts of Bangladesh. These women find it difficult to continue treatment at the centre because they require repeated and frequent visits. More such centres for infertility treatment are in demand in remote areas so that people from all over the country have access to infertility centres of equal and comparable standards.

A study on infertile couples of Ethiopia ${ }^{5}$ revealed 58.1\% of the women were less than 30 years. $38.5 \%$ of women had secondary school education and above, $21.6 \%$ had no formal education and $40.5 \%$ had primary education. Housewives were 70.4\% 87.5\% resided in Addis Ababa, the capital and rural women was only $6.2 \%$. The situation is almost similar to our country except for the large proportion of uneducated women. The fact that both Bangladesh and Ethiopia are developing countries explains the similarity.

Most $(72.72 \%)$ of the women has a monthly family income of less than $20,000 \mathrm{Tk}$. The initial infertility work up in our centre costs 5,800/--10,000/. Tubal evaluation costs 3,000--10,000 Tk on an average. It is apparent from the findings how difficult it is for the women to afford the costs. Financial constraints explains the high drop out from infertility work up in our centre. We should emphasize on seeking and arranging low cost alternatives for women who cannot afford costly investigation and treatment measures.

Most $(60.40 \%)$ of the women has 3-10 yr of infertility, also a $30.30 \%$ of the women has less than 3 yrs of infertility. The probability of achieving a live birth without treatment decrease with increasing age and duration of infertility ${ }^{6}$. Many will conceive eventually without treatment within 3 years. Thereafter the prognosis of success without treatment is relatively less. Nearly one third of the infertile women presenting to us has less than 3 years of infertility. If they cannot afford the expenditure of infertility work up and if they are not in advanced reproductive age, they can be expected to have spontaneous cure for infertility.

Proportion of primary and secondary infertility in our study was $62.94 \%$ and $37.05 \%$. The findings are similar to majority of studies. The western study 3 had $67 \%$ primary infertility and $33 \%$ secondary infertility. The Israeli study ${ }^{7}$ on 2515 couples had primary infertility in $65 \%$ women. An study carried out before on infertile women at Bangabandhu Sheikh Mujib University ${ }^{8}$ also had primary infertility of $61.90 \%$ and secondary infertility of $38 \%$. The Srilankan study ${ }^{4}$ in contrast had higher rate of secondary infertility than primary infertility (160 per 1000 women versus 40.5 women per 1000 women). The Ethiopian study ${ }^{5}$ had a prevalence of primary infertility at $39.4 \%$ and secondary infertility at $60.6 \%$.

In our study puerperal sepsis was the most common $(20.14 \%)$ cause of secondary infertility. The Srilankan study 4 found $11.4 \%$ of women with secondary infertility to have history of puerperal sepsis followed by abortion $(8.1 \%)$.

\section{Conclusion}

In conclusion, a large majority of women visiting our infertility unit are below 30 years, have a monthly family income of less than twenty thousand Taka, are housewives having less than 10 years of education. In addition to identifying and correcting the cause of infertility we should take care to consider the sociodemographic profile of the patients and provide adequate counseling and emotional support and dispel misinformations. There will be many who reject treatment for financial and other reasons or fail treatment. We should guide them to alternatives like adoption or help them come to closure of fertility treatment. The study and its findings should guide us how to address the infertile women's medical, emotional and financial needs effectively and timely.

\section{References}

1. Valsangkar S, Bodhare T, Bele S, Sai S. An evaluation of the effect of infertility on marital, sexual satisfaction indices and health related quality of life in women. 2011; J Hum Reprod and Sci 4(2): 80-85

2. Kazandi M, Guday O, Mirmir TK, Ertuk N, Ozkmay E. The status of depression and anxiety in infertile Turkish couples. Iranian J Reprod Med 2011; 9(2):99-104

3. Donckers J, Evers JLH, Land JA. The long term outcome of 946 consecutive couples visiting a fertility clinic in 2001-2003. 2011; Fertil Steril 96(1): 160-164

4. Jamarakoon S, Rajapaksi L, Seneveratne HR. Prevalence of primary and secondary infertility in Colombo district. The Ceylon J Med Sci 2002 45:83-91

5. Kililla T. Infertility investigation, sociodemographic characteristics and drop out of infertile women at Family Guidance Association of Ethiopia. Ethiop J Health Dev 2011; 14(2):127-134

6. Sundstrom I, Ildgruben A, Hogberg U. Treatment related and treatment independent deliveries among infertile couples, a long term follow up. Acta Obstet Gynecol Scand 1997 ;76:238--242

7. Farhi S, Ben-haroush A. Distribution of causes of infertility in patients attending primary fertility clinics in Israel. IMAJ 2011;13:51-53 8. Akhter S, Alam H, Khanam NN, Zabin F. Characteristics of Infertile couples Mymensingh Med J 2011; 20(1):121-127 